\title{
Detecting Low Frequency Oscillations in Cardiovascular Signals Using Gradient Frequency Neural Networks
}

\author{
Thomas Kaplan ${ }^{1}$ and Elaine Chew ${ }^{2}$ \\ ${ }^{1}$ Queen Mary University of London, London, UK \\ ${ }^{2}$ CNRS - UMR9912 / STMS (IRCAM), Paris, France
}

\begin{abstract}
Gradient Frequency Neural Networks (GFNNs) have been applied successfully to detect pulse and meter (hierarchical groupings of pulses) in complex music audio signals having polyrhythms and syncopation. Here, we apply GFNNs to the detection of low frequency $(L F)$ and high frequency $(H F)$ oscillations in cardiovascular signals, namely the heart rate variations associated with Mayer waves and with respiration, respectively. The cardiovascular time series is treated as music audio for analysis; the electrocardiographic (ECG) signal is processed as a WAV file, and R-R intervals converted to a MIDI file. GFNNs are networks of nonlinear neural oscillators that offer the advantage of high sensitivity at low stimulus amplitudes, compared to linear amplitude responses, for weak signals. The GFNNs entrained with prominent $L F$ peaks at $0.0837 \pm 0.0175 \mathrm{~Hz}$, to $R$ - $R$ intervals of Kundalini meditators from the PhysioBank Exaggerated Heart Rate Oscillations database. When applied to a 15-hour Holter recording of Paroxysmal Atrial Fibrillation, GFNN entrainment showed significant $L F$ activity between $0.04-0.14 \mathrm{~Hz}$, and $H F$ activity at $\sim 0.25 \mathrm{~Hz}$ during sleep. GFNNs present a novel approach to the detection and study of cardiovascular oscillations, inspired by auditory rhythm perception.
\end{abstract}

\section{Introduction}

Gradient Frequency Neural Networks (GFNNs), developed to model the neurodynamics of rhythm perception, have been applied successfully to the tracking of multiscale periodicities in complex musical rhythms. Here, we explore how GFNN models developed to entrain to, and analyse, complex rhythms in musical audio signals might be useful in analysing cardiovascular signals. In particular, we demonstrate the ability for GFNNs to entrain, through prominent oscillator amplitudes, to low frequency oscillations between 0.01 and $0.25 \mathrm{~Hz}$.

In the realm of auditory perception, GFNNs are motivated by evidence that rhythm perception in music has a correlate in entrainment of cortical oscillations [1]. Similarly, there are believed to be oscillatory systems in cerebral hemodynamics. Yücel et al. [2] categorise cardiovascular oscillations in six forms, including: high frequency (HF) oscillations relating to respiration, $\sim 0.30 \mathrm{~Hz}$; low frequency (LF) oscillations relating to muscle tissue of vessels, $\sim 0.1 \mathrm{~Hz}$; and very low frequency (VLF) oscillations relating to nervous activity of vessels, $\sim 0.04 \mathrm{~Hz}$. The LF category is associated with the enigmatic Mayer waves, oscillations in arterial pressure that are distinguishable from respiration and the heartbeat, yet correlated. The heart rate variability (HRV) spectrum consists of a HF band between $0.15-0.40 \mathrm{~Hz}$ and a LF band between $\sim 0.05-0.14 \mathrm{~Hz}$ [3]

Hamner et al. [4] suggested that nonlinear methods may be required to further understand beat-to-beat (R-R interval) oscillations in cardiovascular regulation, as nonlinear interactions might explain the lack of coherence between $\mathrm{R}-\mathrm{R}$ intervals and arterial pressure oscillations. GFNNs are therefore appealing for preliminary experiments involving low frequency cardiovascular oscillations.

The remainder of the paper is organised as follows: Section 2 describes GFNNs, Section 3 describes the data and models we used to produce the results in Section 4. In Section 4.1, we evaluate GFNN entrainment to R-R intervals with exaggerated low frequency oscillations; and, in Section 4.2, we evaluate GFNN entrainment to a 15-hour Holter recording. We conclude with some insights and future work in Section 5.

\section{Gradient Frequency Neural Networks}

GFNNs are oscillating neural networks that process time-varying signals, and describe the dynamics of neural oscillators hypothesised as a correlate of rhythm perception [1]. GFNNs are composed of (layered) arrays of neural oscillators, distributed across a range of natural frequencies (the 'gradient'). Given a stimulus such as an audio signal, the rhythmic frequencies will cause the oscillators to selectively entrain. This can be likened to a linear filter bank; however, each oscillator exhibits nonlinear resonance. Large et al. [5] derived a canonical model where 
each oscillator is based on the Hopf oscillator, configured close to the critical point of Hopf bifurcation. This is an unstable point on the brink of spontaneous oscillation, providing an appealing and extreme sensitivity to weak signals compared to linear filters [1]. This might prove beneficial in processing cardiovascular signals where oscillatory patterns of interest may not be prominent.

The canonical model has a complex valued output $z$, where real and imaginary components denote excitation and inhibition. In the model configuration, there is a linear damping parameter, $\alpha$, frequency detuning parameters, $\delta_{1,2}$, amplitude compressing parameters, $\beta_{1,2}$, and a nonlinearity parameter, $\epsilon$. The model definition is given by

$$
\begin{aligned}
\frac{d z}{d t} & =z\left(\alpha+i \omega+\left(\beta_{1}+i \delta_{1}\right)|z|^{2}\right. \\
& \left.+\frac{\left(\beta_{2}+i \delta_{2}\right) \epsilon|z|^{4}}{1-\epsilon|z|^{2}}\right)+c P(\epsilon, x(t)) A(\epsilon, \bar{z})
\end{aligned}
$$

where $P(\epsilon, x(t))$ and $A(\epsilon, \bar{z})$ respectively denote the passive and activate components of the nonlinear stimulus coupling to a stimulus. The fully expanded definition can be found in [5].

As proposed by Lambert et al. [6], the weighted phase of the GFNN can be calculated as follows:

$$
\Phi=\sum_{i=0}^{N}\left|z_{i}\right| \arg \left(z_{i}\right) .
$$

The weighted phase can be used to illustrate the sensitive response of a GFNN to irregular heartbeats such as ectopics or atrial fibrillation. Figure 1 demonstrates disturbances to the model's weighted phase during the onset of atrial fibrillation, using a segment of R-R intervals from the Physiobank MIT-BIH Arrhythmia Database [7,8] (record 201). This example does not demonstrate any long-term interaction between oscillators and rhythms (entrainment), but simply the immediate effect of successive R-R intervals as stimulus. However, for the remainder of this work, we examine only GFNN entrainment.

\section{Method}

We apply Large et al. [9]'s reference implementation of GFNNs to cardiovascular signals transformed to compatible audio formats: R-R intervals were converted to MIDI pulse trains, and continuous electrocardiographic (ECG) signals were converted to audio WAV files.

As a preliminary investigation, we applied the GFNN to two datasets, namely, (1) the Physiobank Exaggerated Oscillations (ExOs) database [7, 10] and (2) a $\sim 15$ hour $(15 \mathrm{hr} 5 \mathrm{~m} 49 \mathrm{~s})$ proprietary paroxysmal atrial fibrillation (PAF) ECG Holter monitor recording. The ExOs
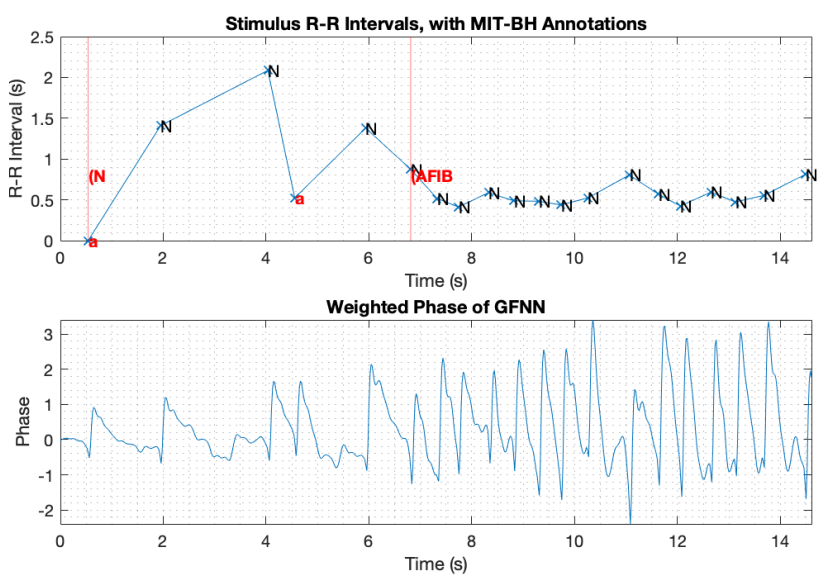

Figure 1. Weighted phase response of GFNN to R-R intervals from record 201 of the MIT-BIH database. Note the irregular and unstable phase response to irregular beats preceding atrial fibrillation (AFIB).

database provided a benchmark in detecting LF oscillations (related to respiration), containing heart rate recordings of advanced Kundalini Yoga meditators ( 2 men, 2 women, mean age of 33 years) in pre-meditation and meditation states. The PAF Holter recording provided a speculative opportunity for evaluating LF and HF oscillations in the presence of PAF.

For both datasets, two-layer networks with local afferent/efferent/internal connections were used. 100 oscillators were used per layer, with a logarithmic gradient of $0.04-0.4 \mathrm{~Hz}$ for the ExOs database and $0.02-0.4 \mathrm{~Hz}$ for the Holter recording. In the first (sensory) layer: $\alpha=$ $1 e^{-5}, \beta_{1}=0, \beta_{2}=-2, \delta_{1}=0, \delta_{2}=0, \epsilon=1$. In the second (motor) layer: $\alpha=-0.4, \beta_{1}=1.75, \beta_{2}=-1.25$, $\delta_{1}=0, \delta_{2}=0, \epsilon=1$. This configuration is similar to that used by Velasco \& Large [11] for pulse detection in syncopated rhythms - rhythms with accents in abnormal time locations-where the second layer is configured to exhibit double-limit-cycle bifurcation behaviour.

This double-limit-cycle oscillator regime is characterised by thresholding and memory; where stable states of oscillation (rest and limit cycle) are separated by an unstable limit cycle, demanding a strong stimulus to cross between the stable states. Memory refers to the fact that the oscillator can remain in the stable limit cycle for longer than the oscillating stimulus. Thresholding refers to the need for a suitably strong oscillating stimulus to activate the stable oscillation. Additionally, the oscillators benefit from high sensitivity: under a low amplitude stimulus they exhibit large amplitude oscillations (a nonlinear response), and sensitivity deteriorates as stimulus amplitude rises (the amplitude curve is compressed). 


\section{4. $\quad$ Results}

Here, we summarise the results of the GFNN on the ExOs dataset and the PAF Holter recording.

\subsection{Exaggerated Oscillations}

In accordance with the observations by Peng et al. [10], we were able to clearly identify low-frequency heart rate oscillations in the advanced meditators through prominent spectral peaks in the $0.025-0.35 \mathrm{~Hz}$ range for the heart rate time series. Figure 2 demonstrates this for one of the Kundalini Yoga meditators, contrasting 300s of the heart rate series during preparatory baseline breathing and then meditation. Notably, this depicts entrainment of the second (motor) layer. Across Kundalini meditators for this period, the frequency at the most prominent peak had a mean of $0.0837 \mathrm{~Hz}$ and standard deviation $0.0175 \mathrm{~Hz}$.
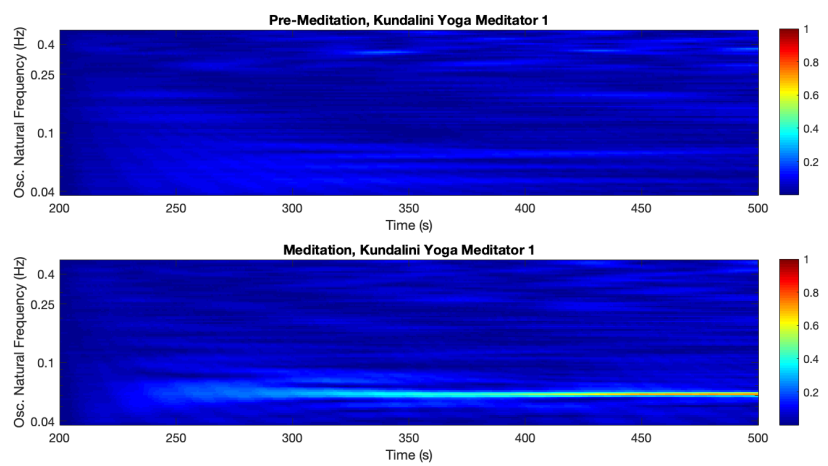

Figure 2. Amplitude spectrum of GFNN motor layer (second layer) from ExOs database for Kundalini Yoga meditator 1. The 200.22 to 499.56-second segment of premeditation and meditation recording is shown. From an early stage of the meditation spectra, a prominent $\sim 0.06 \mathrm{~Hz}$ frequency component can be identified.

Figure 3 demonstrates the final amplitude spectrum of the GFNN across both layers, before and then during meditation for the same meditator. The first (sensory) layer does not exhibit prominent oscillations at the slow breathing pulse frequency.

This reflects the thresholding and memory properties of the double-limit cycle bifurcation, which is configured in the second layer. During preparatory breathing, the second layer exhibited a certain level of noise which would require filtering in some practical application. Existing studies of musical rhythms identify active frequencies using the threshold implicit in oscillatory dynamics under double limit cycle tuning [11], which may form the basis of a strategy to disregard irrelevant oscillatory frequencies in clinical applications.

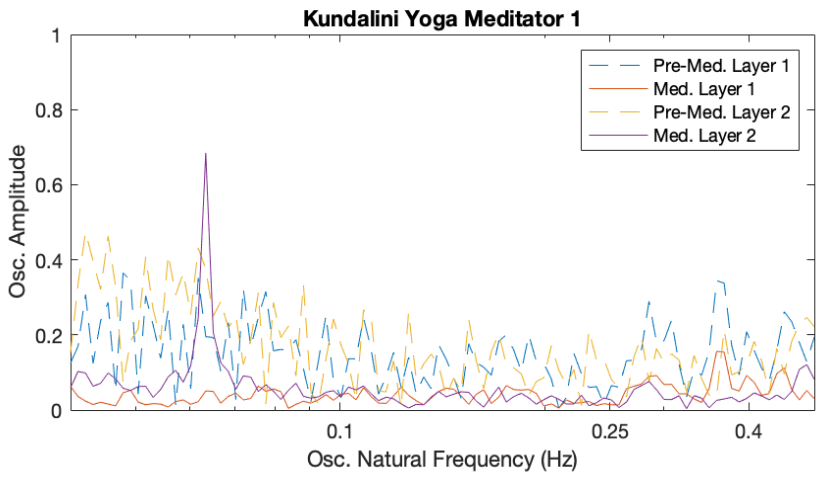

Figure 3. Final GFNN amplitude spectrum for Kundalini Yoga meditator 1 from ExOs database, using the 200.22 to 499.56-second segment of pre-meditation and meditation recording. The second layer during meditation entrains with a prominent frequency component at $\sim 0.06 \mathrm{~Hz}$.

\subsection{Non-Exaggerated Oscillations}

Whereas musical rhythmic patterns or rhythm streams do not typically exceed the order of seconds or minutes, we attempted to assess GFNN entrainment for a 15-hour Holter recording. Unlike the meditative dataset, this afforded an open investigation of the presence of LF and HF oscillations. We extracted an ECG baseline and removed high frequency noise using median filters and 1D linear interpolation.

For the full 15-hour ECG sequence, we ran into issues configuring a single GFNN which would both remain sensitive to weak signals of interest and resilient to transient noise. Despite attempts to configure the amplitude com-

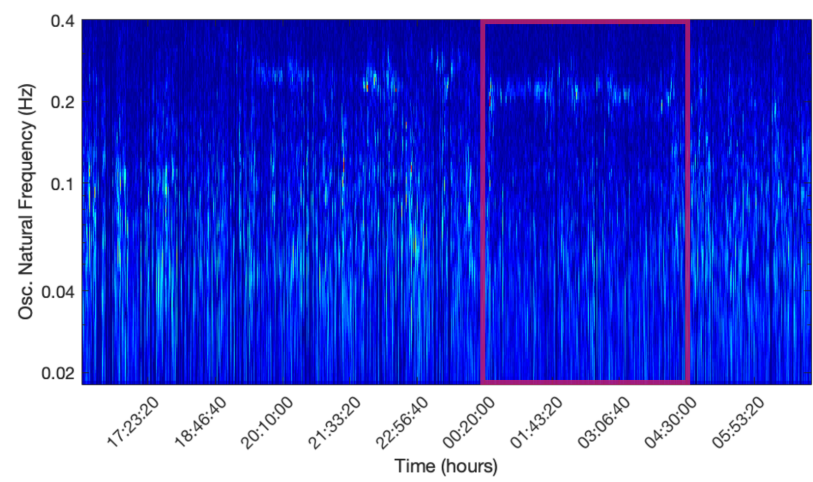

Figure 4. Amplitude spectrum of GFNN motor layer (second layer) for a 15-hour extract of the PAF Holter recording. There is significant activity between $0.02 \mathrm{~Hz}$ and $0.1 \mathrm{~Hz}$, but the most consistent entrainment occurred closer to $\sim 0.25 \mathrm{~Hz}$ between 00:30am and 4:30am (this period is bounded by a red box above). 
pressing parameter, $\alpha$, to improve network stability, transient noise would cause the first layer to reach significant amplitudes and the model would not behave as expected. Therefore, we chose to use windows of 100 seconds with hop sizes of 25 seconds. This is obviously problematic in that GFNN entrainment is lost between intervals, and this drawback will be a high priority focus of future work.

Throughout the recording there was LF activity (between $\sim 0.04-0.14 \mathrm{~Hz}$ ), but it dropped off between 00:30am and 4:30am. During this period, there was a notable rise in HF energy around exactly $0.25 \mathrm{~Hz}$, as shown in Figure 4. The HF component, related to respiration, is sometimes used as an indicator of parasympathetic activity. Sympathetic activity might contribute to the LF component, but LF oscillations remain a contentious measure of sympathetic activity [3]. In future work this LF component might be re-examined in tandem with blood pressure oscillations, after improving long-term GFNN stability.

\section{Conclusion}

We have described GFNNs, a method developed for tracking periodicities in complex musical rhythms, and demonstrated its use in ECG sequences of exaggerated oscillations during meditation and of a 15-hour Holter recording of PAF.

Prominent entrainment to LF and HF heart rate oscillations was achieved by configuring GFNNs to exhibit thresholding, memory, and sensitivity. These properties are largely not exhibited by linear filtering techniques, suggesting nonlinear models of rhythm perception developed for music audio might be useful for cardiovascular signals.

It became evident that future work must carefully consider how these models might be configured for longrunning monitoring of cardiovascular signals, ensuring stability without compromising sensitivity.

GFNNs can also be integrated with other artificial neural networks. Lambert et al. [12] demonstrated that a LongShort Term Recurrent Neural Network (LSTM) could learn long-term entrainment patterns in a GFNN, in order to predict rhythmic events. Hybrid architectures such as these could prove useful in modelling problems outside of music, where the complex interactions between multiple rhythmic signals are evaluated. For example, the interactions between arterial pressure and R-R interval oscillations, as proposed by Hamner et al. [4].

\section{Acknowledgments}

We are grateful to Prof. Pier Lambiase and Prof. Peter Taggart for thought-provoking exchanges on this work. TK is supported by a doctoral studentship from the EPSRC-AHRC Centre for Doctoral Training in Media and Arts Technology (EP/L01632X/1); EC is supported by the
ERC under the EU's Horizon 2020 Research and Innovation programme (Grant agreement No.788960).

\section{References}

[1] Large EW. Neurodynamics of Music. In Riess Jones M, Fay RR, Popper AN (eds.), Music Perception, volume 36. New York, NY: Springer New York. ISBN 978-1-4419-6113-6 978-1-4419-6114-3, 2010; 201-231.

[2] Yücel MA, Selb J, Aasted CM, Lin PY, Borsook D, Becerra L, Boas DA. Mayer waves reduce the accuracy of estimated hemodynamic response functions in functional near-infrared spectroscopy. Biomedical Optics Express July 2016;7(8):3078-3088. ISSN 2156-7085.

[3] Lehrer PM, Gevirtz R. Heart rate variability biofeedback: how and why does it work? Frontiers in Psychology 2014; 5:756. ISSN 1664-1078.

[4] Hamner JW, Morin RJ, Rudolph JL, Taylor JA. Inconsistent link between low-frequency oscillations: R-R interval responses to augmented Mayer waves. Journal of Applied Physiology 2001;90(4):1559-1564.

[5] Large EW, Almonte FV, Velasco MJ. A canonical model for gradient frequency neural networks. Physica D Nonlinear Phenomena June 2010;239(12):905-911. ISSN 0167-2789.

[6] Lambert AJ, Weyde T, Armstrong N. Adaptive Frequency Neural Networks for Dynamic Pulse and Metre Perception. In Proceedings of the International Society for Music Information Retrieval Conference, ISMIR 2016. 2016; 60-66.

[7] Goldberger Ary L., Amaral Luis A. N., Glass Leon, Hausdorff Jeffrey M., Ivanov Plamen Ch., Mark Roger G., Mietus Joseph E., Moody George B., Peng Chung-Kang, Stanley H. Eugene. PhysioBank, PhysioToolkit, and PhysioNet. Circulation June 2000;101(23):e215-e220.

[8] Moody GB, Mark RG. The impact of the MIT-BIH Arrhythmia Database. IEEE Engineering in Medicine and Biology Magazine May 2001;20(3):45-50. ISSN 0739-5175.

[9] Large E. GrFNN Toolbox | Music Dynamics Laboratory, March $2016 . \quad$ URL github.com/MusicDynamicsLab/GrFNNToolbox.

[10] Peng CK, Mietus JE, Liu Y, Khalsa G, Douglas PS, Benson $\mathrm{H}$, Goldberger AL. Exaggerated heart rate oscillations during two meditation techniques. International Journal of Cardiology July 1999;70(2):101-107. ISSN 0167-5273.

[11] Velasco MJ, Large EW. Pulse detection in syncopated rhythms using neural oscillators. In Proc. of the 12th International Society for Music Information Retrieval Conference, ISMIR 2011. Miami, Florida, USA, 2011; 185-190.

[12] Lambert A, Weyde T, Armstrong N. Perceiving and predicting expressive rhythm with recurrent neural networks. In Proceedings of the 12th International Conference in Sound and Music Computing, SMC 2015. Maynooth, Ireland: SMC15. ISBN 978-0-9927466-2-9, January 2015; 8 pages.

Address for correspondence:

Thomas Kaplan

QMUL-EECS, Mile End Rd, London E1 4NS, UK

t.m.kaplan@qmul.ac.uk 